\title{
GRK2 overexpression inhibits IGF1-induced proliferation and migration of human hepatocellular carcinoma cells by downregulating EGR1
}

\author{
YANG MA, CHEN-CHEN HAN, QIONG HUANG, WU-YI SUN and WEI WEI \\ Institute of Clinical Pharmacology, Anhui Medical University, Key Laboratory of Anti-inflammatory and \\ Immune Medicine, Ministry of Education, Hefei, Anhui 230032, P.R. China
}

Received November 19, 2015; Accepted February 1, 2016

DOI: $10.3892 /$ or.2016.4641

\begin{abstract}
G protein-coupled receptor kinase 2 (GRK2) is a serine/threonine kinase that is involved in a variety of important signaling pathways and alternation of GRK2 protein level or activity causes diseases such as heart failure, rheumatoid arthritis, and obesity. However, the role and mechanism of GRK2 in hepatocellular carcinoma (HCC) progression is not fully investigated. In this study we found that GRK2 plays an inhibitory role in IGF1-induced HCC cell proliferation and migration. Overexpression of GRK2 causes a decrease in early growth response-1 (EGR1) expression, while knockdown of GRK2 leads to marked increase in EGR1 expression in the treatment of IGF1. Through co-immunoprecipitation and western blot assay, we confirmed that GRK2 can interact with insulin-like growth factor 1 receptor (IGF-1R) and inhibits IGF1-induced activation of IGF1R signaling pathway. Silencing EGR1 attenuates GRK2 overexpression-caused inhibition of cell proliferation, tumor colony number and migration activity, while overexpressing of EGR1 restores the anti-proliferative and migratory effect by GRK2 overexpression in HCCLM3 cells. Collectively, these results suggest that GRK2 may inhibit IGF1-induced HCC cell growth and migration through downregulation of EGR1 and indicate that enforced GRK2 may offer a potential therapeutic approach against HCC.
\end{abstract}

\section{Introduction}

Hepatocellular carcinoma (HCC) is one of the most common malignant tumors worldwide, especially in Asian countries. It

Correspondence to: Professor Wei Wei, Institute of Clinical Pharmacology, Anhui Medical University, Key Laboratory of Anti-inflammatory and Immune Medicine, Ministry of Education, 81 Meishan Road, Hefei, Anhui 230032, P.R. China

E-mail:wwei@ahmu.edu.cn

Key words: G protein-coupled receptor kinase 2, early growth response-1, insulin-like growth factor 1 receptor signaling pathway, cell proliferation, cell migration accounts for $\sim 70 \%$ of liver cancers and may originate from both liver cirrhosis and non-cirrhotic liver, indicating the existence of different hepatocarcinogenesis pathways (1-3). Although the precise mechanisms may be different according to the different risk factors, the multi-step process of hepatocarcinogenesis may be divided into several phases: chronic liver injury, cell death, cirrhosis and regeneration, DNA damage, dysplasia, and finally HCC (4-6). Through surgical resection and liver transplantation, HCC is potentially curable at early stage. Unfortunately, most of the HCC patients at diagnosis are in the advanced-stage with serious background liver disease which is not suitable for such therapy (7). Moreover, HCC is highly resistant to traditional medical treatment such as radiation and chemotherapy (8). Therefore, the need for the development of effective prevention, and therapy of the disease is urgent.

G protein-coupled receptor kinase 2 (GRK2) is a serine/ threonine protein kinase that is ubiquitously expressed. It was originally found to desensitize $G$ protein coupled receptor (GPCR) signaling by phosphorylating the agonist-occupied receptor $(9,10)$. $\beta$-arrestin then binds to the phosphorylated receptor to form a protein complex which prevents the coupling of the receptor from its G-protein, leading to signaling attenuation (11). Despite of its conventional role as a kinase in receptor desensitization, an increasing number of evidence shows that GRK2 can interact with other transmembrane receptors to participate in various cellular processes, such as receptor tyrosine kinases (RTKs) $(12,13)$. GRK2 translocates to the ligand-activated PDGFR (platelet-derived growth factor receptor) and EGFR (epidermal growth factor receptor), and phosphorylates their intracellular domains. Although the relationship between GRK2 and some human diseases including heart failure, hypertension, rheumatoid arthritis has been intensely characterized $(14,15)$, its role in cancer, particularly in the proliferation of HCC, remains largely unknown.

EGR1, known as early growth response 1 , is a member of the immediate-early gene family. It can be induced by growth factors, cytokines and stress signals such as radiation, damage (16). MAPK families, known as ERK1/2, JNK and p38 MAPK, are most commonly involved in EGR1 activation (17). EGR1 is also a member of zinc-finger transcription factors with an amino terminal activation domain near the carboxyl terminal end of the protein sequence $(18,19)$, so it can bind to 
the promoter of the target gene to regulate the gene expression and be involved in many biological functions, including wound repair, cell growth, migration and invasion (20).

We selected two HCC cell lines, HepG2, with a high expression of GRK2, and HCCLM3, with a low expression of GRK2. To directly study the effect of GRK2 on EGR1 expression, HepG 2 cell line with a functional GRK2 siRNA expression was created, and remarkable elevation in EGR1 expression was observed in the presence of IGF1. HCCLM3 with GRK2 overexpression was created, and remarkable decrease in EGR1 expression was observed in the presence of IGF1. Furthermore, we found that GRK2 negatively regulates IGF1-induced activation of IGF1R signal pathway though binding to IGF1R. This regulation of EGR1 by GRK2 may be essential for HCC cell growth as evidenced by the in vitro cell growth and migration studies. Thus, our data suggest, for the first time, that the regulation of EGR1 by GRK2 is critical for HCC growth and enforced GRK2 may offer a potential therapeutic approach against $\mathrm{HCC}$.

\section{Materials and methods}

Reagent and antibodies. All chemicals and HRP-conjugated anti-rabbit, mouse, goat IgG were purchased from SigmaAldrich. Antibodies against EGR1, phospho-p44/42 MAPK (Erk1/2) (Thr202/Tyr204), p44/42 MAPK (Erk1/2), phosphoAkt (Ser473), Akt, phospho-IGF1R $\beta$ (Tyr1135), IGF1R $\beta$, and human recombinant IGF-1 were from Cell Signaling Technology; antibodies against GRK2, actin and protein A/G plus-agarose were purchased from Santa Cruz Biotechnology.

Cell culture and transfection. Human HCC cell lines, HepG2, SMCC7721, HCCLM3 were maintained in DMEM (Hyclone) supplemented with 10\% FBS (Hyclone) and $2 \mathrm{nmol} / \mathrm{l}$ L-glutamine and penicillin-streptomycin. Cells were cultured in an incubator with humidified air at $37^{\circ} \mathrm{C}$ with $5 \% \mathrm{CO}_{2}$. Lipofectamine-3000 (Invitrogen) was employed for transfection according to the manufacturer's instruction. The stably transfected cell lines were obtained after being selected by $800 \mu \mathrm{g} / \mathrm{ml} \mathrm{G} 418$ for 3-4 weeks. Double-strand siRNAs targeting GRK2, EGR1 and scrambled control was synthesized using the following sequences, siRNA-GRK2; forward, 5'-GCA UCA UGC AUG GCU ACA UdTdT, reverse, 5'-AUG UAG CCA UGC AUG AUG CdTdT; siRNA-EGR1; forward, 5'-CCU GGA GCC UGC ACC CAA CdTdT, reverse, 5'-GUU GGG UGC AGG CTC CAG GdTdT, siRNA-scramble: forward 5'-AUG AAC GUG AAU UGC UCA AdTdT, reverse 5'-UUG AGC AAU UCA CGU UCA UdTdT. SiRNA duplexes were purchased from GenePharma.

RT-PCR and real-time PCR. Total RNA was isolated by TRIzol (Invitrogen) according to the manufacturer's instructions, and $1 \mu \mathrm{g}$ RNA was converted to cDNA using the high capacity cDNA reverse transcription kits (Invitrogen). The following primer sets were used for RT-PCR and real-time PCR: ACTIN-Fw: 5'-GAC CTG ACT GAC TAC CTC ATG AAG AT-3', ACTIN-Re: 5'-GTC ACA CTT CAT GAT GGA GTT GAA GG-3'; EGR1-Fw: 5'-CTG ACC GCA GAG TCT TTT CCT G-3', EGR1-Re: 5'-TGG GTG CCG CTG AGT AAA TG-3'; GRK2-Fw: 5'-GTT GCT GCA GAG GGA TGT
CAA CCG-3', GRK2-Re: 5'-GTC AGG AAG GGG TTG CCC ATC TTG G-3'.

Immunoprecipitation. Cells were washed with ice-cold PBS and lysed in $800 \mu \mathrm{l} \mathrm{NP}-40$ solubilization buffer for $0.5 \mathrm{~h}$. The lysate was centrifuged and the supernatant was incubated with $1 \mu \mathrm{g}$ of anti-IGF1R antibody and $15 \mu \mathrm{l}$ of $50 \%$ slurry of protein $\mathrm{A} / \mathrm{G}$ plus-agarose beads at $4^{\circ} \mathrm{C}$ overnight. The beads were subsequently washed, and the proteins bound to the beads were separated by SDS-PAGE. The samples were detected in the subsequent western blot with anti-GRK2 and anti-IGF1R antibody.

Western blot analysis. Cells were harvested and lysed in the RIPA lysis buffer to prepare the whole cell extract. Protein concentration was determined using Bradford assay (Bio-Rad, Hercules, CA, USA). Lysates were subjected to SDS-PAGE and immunoblot analysis. Horseradish peroxidase-conjugated secondary antibodies and enhanced chemiluminescence detection (ImageQuant) was used to detect specific immunoreactive proteins.

Cell proliferation assays. The proliferation of HCC cells were examined using Cell Counting Kit 8 (Dojindo) according to the manufacturer's instructions. Equal numbers of cells in a volume of $100 \mu \mathrm{l}$ were seeded in a 96-well plates. The cells were transduced with GRK2 expression plasmid, GRK2 siRNA duplex and their corresponding controls with and without IGF1 stimulation. The plates were incubated for 3 days. Cell proliferation was determined every 24 h. For each measurement, $10 \mu \mathrm{l} \mathrm{CCK}$ was added into each well and incubated at $37^{\circ} \mathrm{C}$ with $5 \% \mathrm{CO}_{2}$ for $1 \mathrm{~h}$. The absorbance of the plate was taken at $450 \mathrm{~nm}$ in an ELISA plate reader. All assays were done in triplicate.

Tumor colony formation assay. After the stable transfected HCCLM3/pc-GRK2 and its control cells were transfected with EGR1 siRNA or scrambled siRNA for 48 h, 5000 cells were suspended in $1.5 \mathrm{ml}$ DMEM with $0.35 \%$ agar, $50 \mathrm{ng} / \mathrm{ml} \mathrm{IGF1}$ and $5 \%$ fetal bovine serum and seeded in 6-well plates which were pre-coated with the same medium except containing $0.5 \%$ agar. The cells were allowed to form colonies for 2 weeks. The colonies were stained with $0.1 \%$ crystal violet for $10 \mathrm{~min}$ at $37^{\circ} \mathrm{C}$ and counted.

Wound healing assay. HepG2 and HCCLM3 cells transfected with corresponding plasmids or siRNA were seeded into 24-well plates, at 80-90\% confluency, the cell monolayer was disrupted with a cell scraper. After washing with PBS three times to remove cell debris, the remaining cells were treated with $50 \mathrm{ng} / \mathrm{ml}$ IGF1 and then images were captured by microscope at 0 and $24 \mathrm{~h}$ after treatment. Cell motility was evaluated according to the following formula: Cell motility $=$ (distance $24 \mathrm{~h}$ - distance $0 \mathrm{~h}$ )/distance $0 \mathrm{~h}$.

Statistical analysis. All experiments were performed in triplicates. The data represent the mean \pm SD. Statistical analysis was performed with SPSS 17.0 software. Student's T-test was used to analyze the significance between two groups. Statistical significance was set at $\mathrm{P}<0.05, \mathrm{P}<0.01$. 

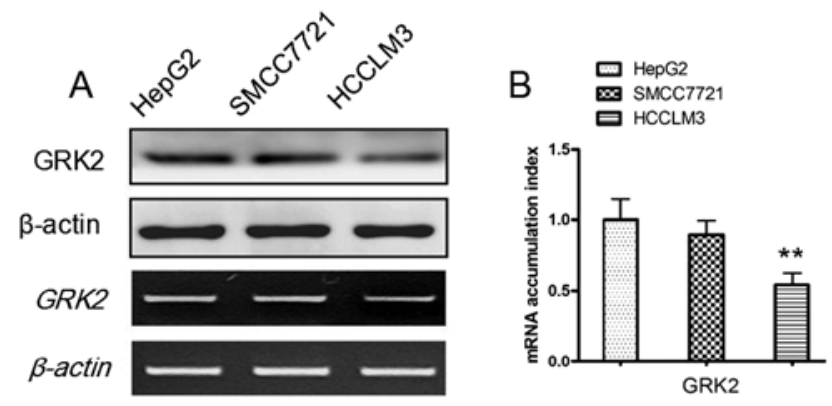
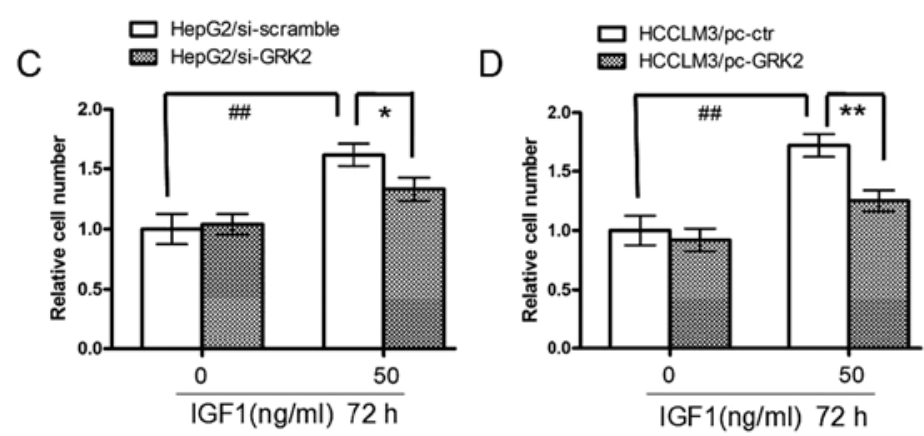

E

$\mathrm{Oh}$
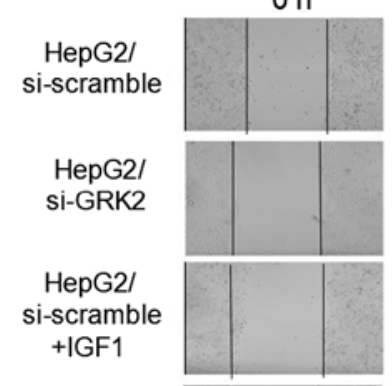

HepG2/

si-GRK2

+IGF1

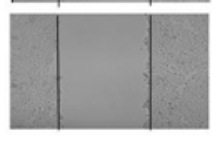

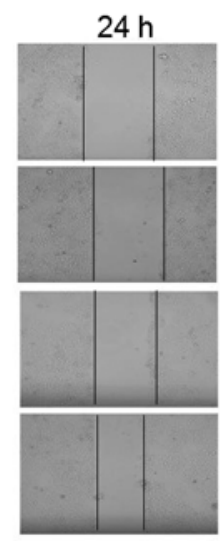
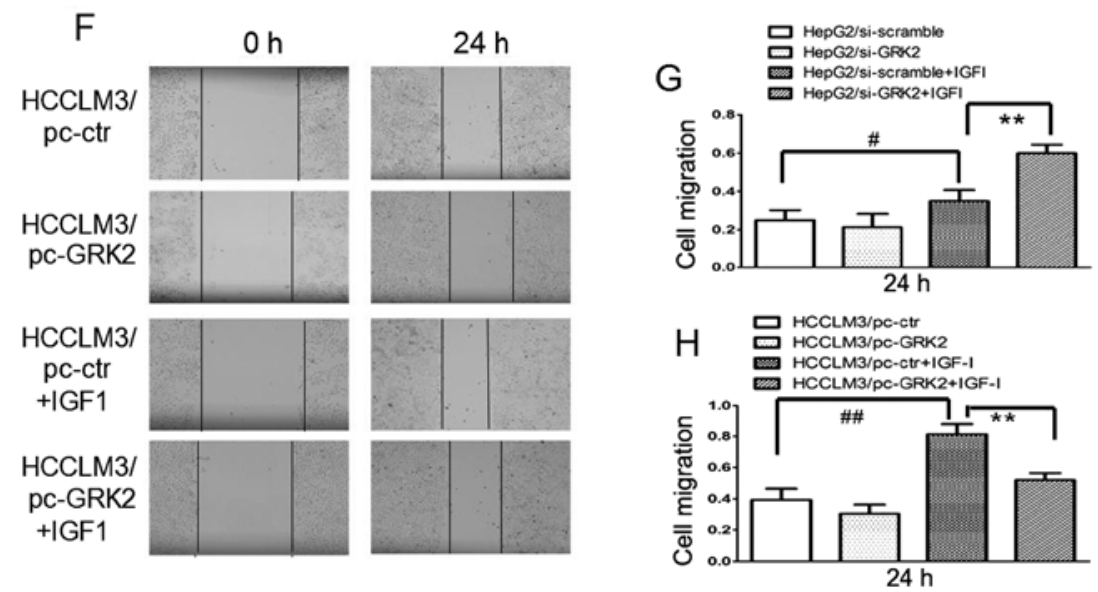

Figure 1. The regulation of IGF1-induced human HCC cell proliferation and migration by GRK2. (A and B) GRK2 levels were evaluated on three HCC cell lines, HepG2, SMCC7721, HCCLM3 by western blotting, RT-PCR and real-time PCR ( ${ }^{* *}$ P $<0.01$ compared with HepG2). (C and D) Growth of HepG2/ si-scramble, HepG2/si-GRK2 and HCCLM3/pc-ctr, HCCLM3/pc-GRK2 cells treated with $50 \mathrm{ng} / \mathrm{ml}$ IGF1 or the vehicle (0.1\% DMSO) for $72 \mathrm{~h}$. (E-H) Woundhealing analysis of HCC cell migration for 0 and $24 \mathrm{~h}$, images and quantitative analysis of HepG2/si-scramble, HepG2/si-GRK2 and HCCLM3/pc-ctr, HCCLM3/pc-GRK2 cells treated with $50 \mathrm{ng} / \mathrm{ml}$ IGF1 or the vehicle at 0 and $24 \mathrm{~h} ;{ }^{\# \#} \mathrm{P}<0.01,{ }^{\#} \mathrm{P}<0.05$ compared with ctr group, ${ }^{* * *} \mathrm{P}<0.01,{ }^{*} \mathrm{P}<0.05$ compared with ctr+IGF1 group).

\section{Results}

Regulation of IGFI-induced human HCC cell proliferation and migration by GRK2. A recent study indicates that GRK2 has an inhibitory role in regulating IGF1-stimulated signaling pathway in HEK293T cells (21). This observation promoted us to investigate whether GRK2 is critical for IGF1-induced human HCC cell proliferation and migration. To gain a profile of basal GRK2 levels in HCC cells, we examined GRK2 expression in three HCC cell lines by western blot and RT-PCR analysis, with the highest level in the HepG2 cell line and the lowest level in the HCCLM3 cell line (Fig. 1A and B). We thus selected HepG2 for GRK2 knockdown experiments (HepG2/si-GRK2) and HCCLM3 cell lines for GRK2 overexpression (HCCLM3/pc-GRK2) experiments. As shown in Fig. 1C, E and G, suppression of GRK2 in HepG2 led to an increase in IGF1-induced cell proliferation and migration compared with control cells. While in HCCLM3, as shown in Fig. 1D, $\mathrm{F}$ and $\mathrm{H}$, overexpression of GRK2 led to a decrease in IGF1-induced cell proliferation and migration. These results indicate that GRK2 can negatively regulate IGF1-induced tumor cell growth. However, GRK2 alone can not affect HCC cell growth without IGF1 stimulation (Fig. 1C-H).

GRK2 induces downregulation of EGR1 expression in the IGF1 treatment. To determine the mechanisms of proliferation inhibition role of GRK2, we next investigated GRK2 regulated genes in HCC cells. From unpublished microarray results, we found that EGR1 expression can be inhibited by GRK2 overexpression in the presence of IGF1. As shown in Fig. 2A-C, significant increases of EGR1 levels were observed in GRK2 siRNA cells compared with the control cell as evidenced by RT-PCR, real-time PCR and western blot experiments. In contrast to GRK2 downregulation, overexpression of GRK2 in HCCLM3 led to a decrease in EGR1 protein and mRNA levels (Fig. 2D-F). These results revealed that GRK2 induced downregulation of the EGR1 expression in the IGF1 treatment of HCC cell lines.

GRK2 negatively regulates the IGF1-induced IGFIR signaling pathway through interacting with IGFIR. Previous studies indicated that activated IGF-1R can stimulate the downstream Erk signaling to ultimately transactivate the EGR1 transcription factor $(22,25)$. In order to explore the mechanisms by which GRK2 regulates the EGR1 expression, we investigated the influence of ectopic expression of GRK2 on IGF1 signaling pathways, and analyzed the IGF1R, ERK and AKT activation in HCC cells expressing high levels of GRK2, or a siRNA directed against GRK2. As shown in Fig. 3A and B, silencing of GRK2 led to an increase in IGF1-induced activation of IGF1R, ERK and AKT in HepG2 cells. Conversely, in Fig. 3C and D, overexpression of GRK2 
A
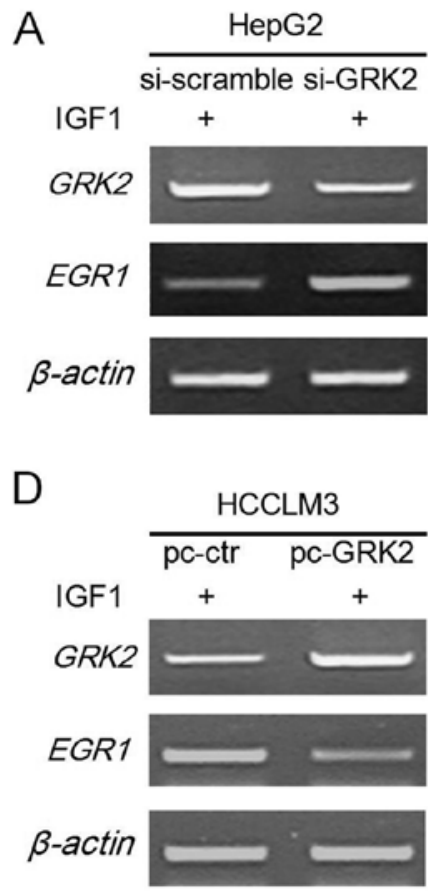

B

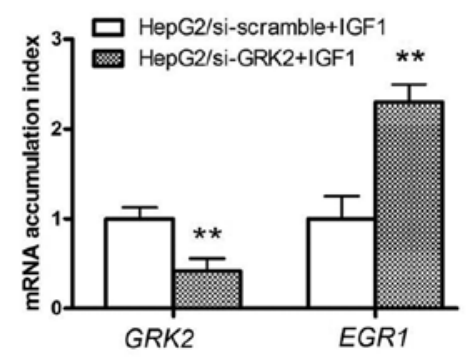

E

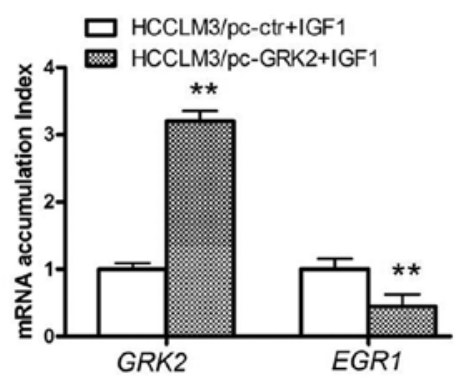

C

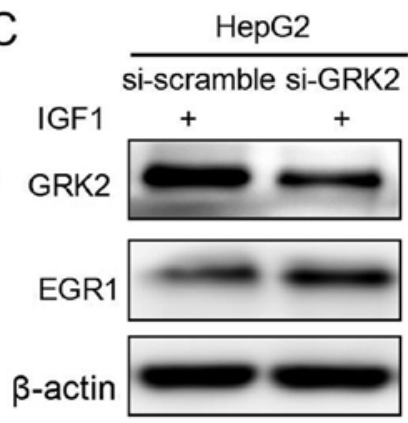

$\mathrm{F}$ HCCLM3

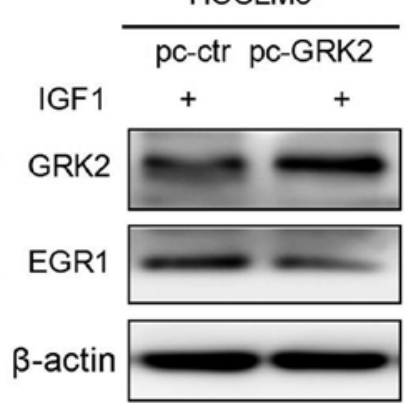

Figure 2. GRK2 induces downregulation of EGR1 expression in the treatment of IGF1. (A-C) The expression of GRK2 and EGR1 in HepG2/si-scramble and HepG2/si-GRK2 cells treated with $50 \mathrm{ng} / \mathrm{ml}$ IGF1 was analyzed by western blotting, real-time PCR and RT-PCR. $\left({ }^{* *} \mathrm{P}<0.01\right.$ compared with HepG2/siscramble+IGF1 group). (D-F) The expression of GRK2 and EGR1 in HCCLM3/pc-ctr and HCCLM3/pc-GRK2 cells treated with $50 \mathrm{ng} / \mathrm{ml}$ IGF1 was analyzed by western blotting, real-time PCR and RT-PCR; ( ${ }^{* *} \mathrm{P}<0.01$ compared with HCCLM3/pc-ctr+IGF1).

caused a decrease in IGF1-induced IGF1R, ERK and AKT activation in HCCLM3 cells.

GRK2 can desensitize GPCR signaling and some tyrosine kinase receptors by binding to the agonist-occupied receptor $(9,10)$. Then we analyzed the relationship between GRK2 and IGF1R in an HCC cell line. The association of GRK2 and IGF1R was observed in the IGF1 treatment of HCCLM3 cells, and after GRK2 overexpression, this association was increased in the stimulated conditions (Fig. 3E), suggesting that GRK2 overexpression increased the formation of GRK2IGF1R complex in a ligand-dependent manner. These results demonstrated that overexpression of the GRK2 suppressed IGF1R signaling pathway through interacting with IGF1R, and this regulation may lead to decreased EGR1 expression.

Anti-proliferative and migratory effect by overexpression of GRK2 is EGR1-mediated. Since GRK2 has been involved in HCC cell proliferation and migration, we investigated whether EGR1 is critical for GRK2-regulation of these functions. Stably transfected HCCLM3/pc-GRK2 and HCCLM3/pc-ctr cells were transiently transfected with either siRNA-EGR1 or EGR1 expression plasmid, the cell scratch migration (Fig. 4A and $\mathrm{C}$ ), tumor colony-forming (Fig. 4B and D) and proliferation assays (Fig. 4E) were performed with the cells in the presence of IGF1. Overexpression of GRK2 or suppression of EGR1 in HCCLM3/pc-ctr cells led to significant decrease in cell proliferation, colony growth and migration compared to control cells. Of note, treatment with EGR1 siRNA minimized the difference in cell growth and migration between HCCLM3/pc-GRK2 cells and control cells, while overexpression of EGR1 restored the anti-proliferative and migratory effect by GRK2 overexpression in HCCLM3 cells.
These results indicated that GRK2 inhibits IGF1-induced HCC cell growth and migration through downregulating EGR1.

\section{Discussion}

GRK2 is known to have antiproliferative, antitumor activities in some tumors. Enforced overexpression of GRK2 inhibited cell proliferation in human thyroid cancer cells and in human smooth muscle cells $(23,24)$; however, the mechanisms by which the proliferation activity was mediated by GRK2 is not clearly understood. In this study, we illustrated a novel mechanism that enforced GRK2 blocks HCC cell proliferation and migration induced by IGF1, including repressing EGR1 expression and blocking the IGF1R signaling pathway by interacting with IGF1R. This is the first report of GRK2's involvement in the regulation of EGR1 expression.

In this study, GRK2 overexpression alone was not able to suppress the proliferation of HCCLM3 cells in the absence of IGF1, which seems to contradict the published data that enforced GRK2 by adenovirus transduction inhibited HCC cell growth (24). One explanation may be the differences of cell types, Mahlavu and Huh7 cells were chosen in the previous study, whereas HCCLM3 and HepG2 were used in the current study. Another possible explanation is that the time lengths in the proliferation assay are different, in the previous study the test time was four days, and GRK2 inhibited the growth of Mahlavu and Huh7 cells on the third and fourth day, but there is no difference of cell growth in the first two days. In the current study, we tested three days of cell proliferation, and did not detect the difference during this period.

While EGR1 is crucial for the proliferation of hepatocytes and plays an important role in liver regeneration (26), the 

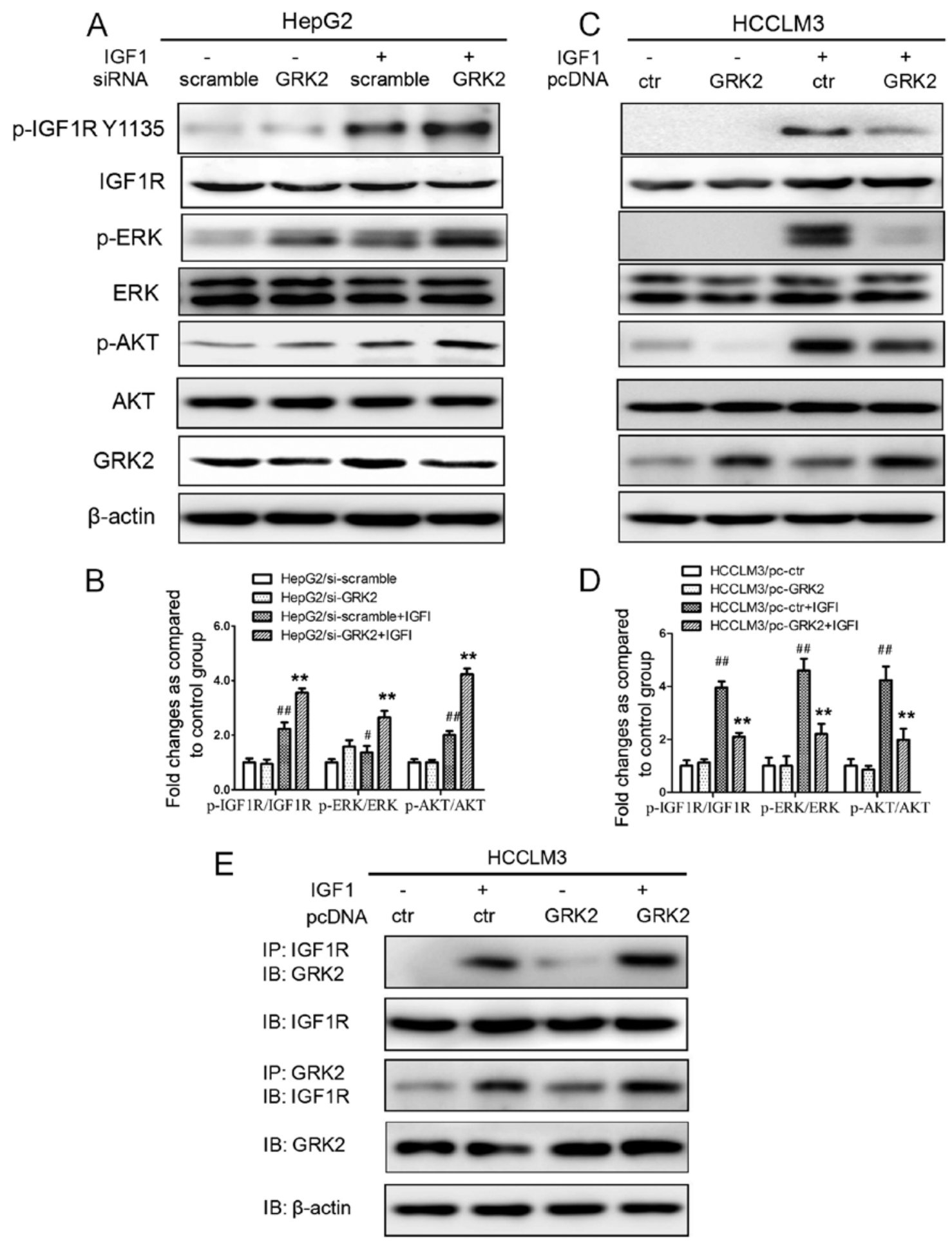

Figure 3. GRK2 negatively regulates the IGF1-induced IGF1R signaling pathway through interacting with IGF1R. (A and B) Whole cell lysates from HepG2/ si-GRK2, HCCLM3/pc-GRK2 cells and the control cells which were treated with $50 \mathrm{ng} / \mathrm{ml}$ IGF1 or the vehicle were subjected to SDS-PAGE analyses for the expression of GRK2, IGF1R, ERK and AKT. Activation of IGF1R, Erk and AKT was examined by immunoblot assays using anti-phosphorylated antibody. (C and D) The histogram corresponding to the quantitative analysis of IGF1R, Erk and AKT activation are shown. The band intensities of phosphorylation of IGF1R, Erk and AKT were normalized to that of the corresponding non-phosphorylation. The data from three independent experiments are shown as the mean \pm SD. Densitometry values in the histograms were expressed as fold-change relative to the normal control, ${ }^{\# \#} \mathrm{P}<0.01$ compared with normal control group ${ }^{* *} \mathrm{P}<0.01$ compared with control plus IGF1 group. (E) HCCLM3 cells were either transfected with control or GRK2 expressing plasmid, serum starved for $12 \mathrm{~h}$, and stimulated or not with IGF1 $(50 \mathrm{ng} / \mathrm{ml})$ for $10 \mathrm{~min}$. The cell lysates were immunoprecipitated with anti-IGF1R or GRK2 antibody, followed by immunoblotting for GRK2 or IGF1R. $\beta$-actin levels in the total protein lysates before IP were used as loading controls.

expression level of EGR1 and its impact in HCC are controversial. One study found that EGR1 is overexpressed in HCC (27), while another study found downregulation of EGR1 expression (28). Also studies have indicated that increased HGF/c-Met signaling could lead to growth-promoting functions of EGR1 in HCC $(29,30)$. In our study, the growth- 



Figure 4. The anti-proliferative and migratory effect by overexpression of GRK2 is EGR1-mediated. (A and C) Wound-healing analysis of HCCLM3 cell migration, images and quantitative analysis of HCCLM3/pc-ctr and HCCLM3/pc-GRK2 cells transfected with EGR1 siRNA or EGR1 expression plasmid in the IGF1 treatment at 0 and $24 \mathrm{~h}$. (B and D) Tumor colony formation of HCCLM3/pc-ctr and HCCLM3/pc-GRK2 cells transfected with EGR1 siRNA or EGR1 expression plasmid in the IGF1 treatment. Cell colonies were visualized under a microscope (B) and the number was counted and presented as number/well (D). (E) Growth of HCCLM3/pc-ctr and HCCLM3/pc-GRK2 cells transfected with EGR1 siRNA or EGR1 expression plasmid in the presence of $50 \mathrm{ng} / \mathrm{ml}$ IGF1 for $72 \mathrm{~h}\left({ }^{* *} \mathrm{P}<0.01\right.$ compared with HCCLM3/pc-ctr+si-scramble group, ${ }^{\# \#} \mathrm{P}<0.01$ compared with HCCLM3/pc-GRK2+si-scramble group). (F) Schematic model of the GRK2 proliferation and migration inhibition. Overexpression of GRK2 inhibits IGF1R signaling activation through increasing the formation of GRK2-IGF1R complex in a ligand-dependent manner, prevents the activation of PI3K/AKT and MEK/ERK pathway, and inhibits expression of Egr-1, resulting in suppression of proliferation and migration of HCC.

promoting effect of EGR1 may arise due to increased MEK/ ERK and PI3K/AKT signaling by IGF1. Many studies show that transcription factor EGR1 is a key regulator in mediating HGF-induced genes that encode regulators of cell proliferation, migration, invasion, and metastasis (29-31).

Taken together, these observations presented in this study indicated that GRK2 plays regulatory functions in HCC cell growth. They also suggested that enforced GRK2 may offer a potential therapeutic approach in the treatment of HCC.

\section{Acknowledgements}

This study was supported by National Natural Science Foundation of China (no. 81502123; 81330081; 81202596), Natural Science Foundation of Anhui Province (no. 1308085QH130), Anhui Province Nature Science Foundation in University (no. KJ2014A119), Grants for Scientific Research of BSKY from Anhui Medical University (no. XJ201212), Specialized Research Fund for the Doctoral Program of Higher Education (no. 20113420120006; 20123420110003), Program for tackling key problems in science and technology by Anhui Province (no. 1301042098).

\section{References}

1. Geisler F and Strazzabosco M: Emerging roles of Notch signaling in liver disease. Hepatology 61: 382-392, 2015.

2. Ding J and Wang H: Multiple interactive factors in hepatocarcinogenesis. Cancer Lett 346: 17-23, 2014.

3. Huang J, Xu L, Luo Y, He F, Zhang Y and Chen M: The inflammation-based scores to predict prognosis of patients with HCC after hepatectomy. Med Oncol 31: 883, 2014.

4. Coulouarn C and Clément B: Stellate cells and the development of liver cancer: Therapeutic potential of targeting the stroma. J Hepatol 60: 1306-1309, 2014.

5. Cervello M, McCubrey JA, Cusimano A, Lampiasi N, Azzolina A and Montalto G: Targeted therapy for HCC: Novel agents on the horizon. Oncotarget 3: 236-260, 2012.

6. Stauffer JK, Scarzello AJ, Jiang Q and Wiltrout RH: Chronic inflammation, immune escape, and oncogenesis in the liver: A unique neighborhood for novel intersections. Hepatology 56: 1567-1574, 2012.

7. Bruix J and Sherman M; Practice Guidelines Committee, American Association for the Study of Liver Diseases: Management of hepatocellular carcinoma. Hepatology 42: 1208-1236, 2005.

8. Cheng AL, Kang YK, He AR, Lim HY, Ryoo BY, Hung CH, Sheen IS, Izumi N, Austin T, Wang Q, et al: Safety and efficacy of tigatuzumab plus sorafenib as first-line therapy in subjects with advanced hepatocellular carcinoma: a phase 2 randomized study. J Hepatol 63: 896-904, 2015. 
9. Tutunea-Fatan E, Caetano FA, Gros R and Ferguson SS: GRK2 targeted knock-down results in spontaneous hypertension, and altered vascular GPCR signaling. J Biol Chem 290: 5141-5155, 2015.

10. Penela P, Ribas C, Aymerich I, Eijkelkamp N, Barreiro O, Heijnen CJ, Kavelaars A, Sánchez-Madrid F and Mayor F Jr: $\mathrm{G}$ protein-coupled receptor kinase 2 positively regulates epithelial cell migration. EMBO J 27: 1206-1218, 2008.

11. Ma L and Pei G: $\beta$-arrestin signaling and regulation of transcription. J Cell Sci 120: 213-218, 2007.

12. Guo J, Chen H, Ho J, Mancini J, Sontag T, Laporte SA, Richard DE and Lebrun JJ: TGFbeta-induced GRK2 expression attenuates AngII-regulated vascular smooth muscle cell proliferation and migration. Cell Signal 21: 899-905, 2009.

13. Jiang $X$, Yang $P$ and Ma L: Kinase activity-independent regulation of cyclin pathway by GRK2 is essential for zebrafish early development. Proc Natl Acad Sci USA 106: 10183-10188, 2009.

14. Avendaño MS, Lucas E, Jurado-Pueyo M, Martínez-Revelles S, Vila-Bedmar R, Mayor F Jr, Salaices M, Briones AM and Murga C: Increased nitric oxide bioavailability in adult GRK2 hemizygous mice protects against angiotensin II-induced hypertension. Hypertension 63: 369-375, 2014.

15. Lymperopoulos A, Rengo G, Funakoshi H, Eckhart AD and Koch WJ: Adrenal GRK2 upregulation mediates sympathetic overdrive in heart failure. Nat Med 13: 315-323, 2007.

16. Liang XH, Deng WB, Li M, Zhao ZA, Wang TS, Feng XH, Cao YJ, Duan EK and Yang ZM: Egrl protein acts downstream of estrogen-leukemia inhibitory factor (LIF)-STAT3 pathway and plays a role during implantation through targeting Wnt4. J Biol Chem 289: 23534-23545, 2014.

17. Chu L, Wang T, Hu Y, Gu Y, Su Z and Jiang H: Activation of Egr-1 in human lung epithelial cells exposed to silica through MAPKs signaling pathways. PLoS One 8: e68943, 2013.

18. Grotegut S, von Schweinitz D, Christofori G and Lehembre F Hepatocyte growth factor induces cell scattering through MAPK/Egr-1-mediated upregulation of Snail. EMBO J 25: 3534-3545, 2006.

19. Ma Y, Cheng Q, Ren Z, Xu L, Zhao Y, Sun J, Hu S and Xiao W: Induction of IGF-1R expression by EGR-1 facilitates the growth of prostate cancer cells. Cancer Lett 317: 150-156, 2012.

20. Ma J, Ren Z, Ma Y, Xu L, Zhao Y, Zheng C, Fang Y, Xue T, Sun B and Xiao W: Targeted knockdown of EGR-1 inhibits IL-8 production and IL-8-mediated invasion of prostate cancer cells through suppressing EGR-1/NF-kappaB synergy. J Biol Chem 284: 34600-34606, 2009 .
21. Zheng H, Worrall C, Shen H, Issad T, Seregard S, Girnita A and Girnita L: Selective recruitment of G protein-coupled receptor kinases (GRKs) controls signaling of the insulin-like growth factor 1 receptor. Proc Natl Acad Sci USA 109: 7055-7060, 2012.

22. Peppel K, Jacobson A, Huang X, Murray JP, Oppermann M and Freedman NJ: Overexpression of $\mathrm{G}$ protein-coupled receptor kinase-2 in smooth muscle cells attenuates mitogenic signaling via $\mathrm{G}$ protein-coupled and platelet-derived grow th factor receptors. Circulation 102: 793-799, 2000.

23. Métayé T, Levillain P, Kraimps JL and Perdrisot R: Immunohistochemical detection, regulation and antiproliferative function of G-protein-coupled receptor kinase 2 in thyroid carcinomas. J Endocrinol 198: 101-110, 2008.

24. Wei Z, Hurtt R, Gu T, Bodzin AS, Koch WJ and Doria C: GRK2 negatively regulates IGF-1R signaling pathway and cyclins' expression in HepG2 cells. J Cell Physiol 228: 1897-1901, 2013.

25. Criswell T, Beman M, Araki S, Leskov K, Cataldo E, Mayo LD and Boothman DA: Delayed activation of insulin-like growth factor-1 receptor/Src/MAPK/Egr-1 signaling regulates clusterin expression, a pro-survival factor. J Biol Chem 280: 14212-14221, 2005.

26. Mueller L, Broering DC, Meyer J, Vashist Y, Goettsche J, Wilms $\mathrm{C}$ and Rogiers $\mathrm{X}$ : The induction of the immediate-earlygenes Egr-1, PAI-1 and PRL-1 during liver regeneration in surgical models is related to increased portal flow. J Hepatol 37: 606-612, 2002.

27. Kawashita Y, Ohtsuru A, Kaneda Y, Nagayama Y, Kawazoe Y, Eguchi S, Kuroda H, Fujioka H, Ito M, Kanematsu T, et al: Regression of HCC in vitro and in vivo by radiosensitizing suicide gene therapy under the inducible and spatial control of radiation. Hum Gene Ther 10: 1509-1519, 1999.

28. Hao MW, Liang YR, Liu YF, Liu L, Wu MY and Yang HX: Transcription factor EGR1 inhibits growth of HCC and esophageal carcinoma cell lines. World J Gastroenterol 2: 203-207, 2002.

29. Lee KH and Kim JR: Hepatocyte growth factor induced up-regulations of VEGF through Egr-1 in hepatocellular carcinoma cells. Clin Exp Metastasis 26: 685-692, 2009.

30. Ozen E, Gozukizil A, Erdal E, Uren A, Bottaro DP and Atabey N: Heparin inhibits Hepatocyte Growth Factor induced motility and invasion of hepatocellular carcinoma cells through early growth response protein 1. PLoS One 7: e42717, 2012.

31. Shin SY, Kim JH, Baker A, Lim Y and Lee YH: Transcription factor Egr-1 is essential for maximal matrix metalloproteinase-9 transcription by tumor necrosis factor alpha. Mol Cancer Res 8: 507-519, 2010. 